\title{
USING THE FUZZY TIME SERIES TECHNIQUE TO IMPROVE THE ESTIMATION OF THE SPECTRAL DENSITY FUNCTION
}

\author{
${ }^{1}$ Department of Mathematics \\ Faculty of Science \\ Tanta University \\ Tanta \\ Egypt \\ e-mail: teamah4@hotmail.com \\ 2Department of Mathematics \\ Faculty of Science \\ Al-Azhar University (Girls) \\ Nasr-City, Cairo \\ Egypt \\ ${ }^{3}$ Department of Mathematics \\ Faculty of Science \\ Al-Azhar University (Men) \\ Nasr-City, Cairo \\ Egypt \\ e-mail: mwa_t95@yahoo.com
}

ABD EL-MONEIM A. M. TEAMAH ${ }^{1}$, HASNAA M. FAIED ${ }^{2}$ and MOHAMMED H. EL-MENSHAWY ${ }^{3}$

2010 Mathematics Subject Classification: 62M10.

Keywords and phrases: fuzzy time series, fuzzy observations, spectral density function, periodogram, estimation.

Received June 9, 2018; Revised July 30, 2018 


\begin{abstract}
In this paper, we study the estimation of the spectral density function and properties of the resulting estimator which called the periodogram. The statistical properties for this periodogram in case of actual observations and forecasted observations of the fuzzy time series are studied. Depending on MSEs which based on these statistical properties, the periodogram results can be compared in both cases. For this purpose a program that transfer the observed time series to fuzzy time series with large sizes is constructed.
\end{abstract}

\title{
1. Introduction
}

In physical sciences experimentalists often measure quantities with respect to an independent variable that is ordered such as time, depth or distance along a line. Time series analysis is the study of the statistical properties of such ordered measurements. The spectral analysis is an important technique in time series analysis. This technique can be of value in understanding physical and other kinds of data. The spectral analysis of time series is one of the oldest and most widely used techniques in the physical sciences. The basic idea behind spectral analysis is to decompose the variance (covariance) of a time series into a number of components. Each components can be associated with a particular frequency. Although the basic ideas and concepts behind spectral analysis are quite old two early references are Stokes [1] and Schuster [2]. The current wide spread use and interest in the subject arose because of three important events in late 1950 and mid 1960 the publication of the influential exposition by Blackman and Tukey [3]; the rediscover of the fast Fourier transform algorithm Cooley and Tukey [4]; and the increasing availability of powerful electronic computers which allowed practitioners to carry out the necessary computations. Spectral analysis is an industry standard in the physical sciences and can be readily done on general purpose computers using software in a variety of forms. As early as the late $19^{\text {th }}$ century, Lee [5] introduced the periodogram which may be regarded as the origin of spectral analysis. There are many improvements for this periodogram by addition taper or 
multitaper for this periodogram, also there are many of statistical studies and analytical conducted on this side (see Anderson [7] and Teamah [8], [9], [10], [11], [12], and [13]). The latter technique is a classical nonparametric tool for analyzing time series. Although the periodogram is an asymptotically unbiased estimate of the spectral density of an underlying stationary process, it is not consistent. Parzen [6] concerned with the spectral analysis of wide sense stationary time series which possess a spectral density function and whose fourth moment functions satisfy an inegrability condition (which includes Gaussian process). Here we have actual observations in our life which is a realizations of a process but it is not easy to find it, and seek to find spectral density function estimator which called the periodogram. By using Chen [17], the actual observations can be transformed into fuzzy observations and then to forecasted observations which carry numerical values. The statistical properties for this periodogram in case of actual observations and forecasted observations are studied. Depending on MSEs which based on these statistical properties, the periodogram results can be compared in case of actual observation and forecasted observations. We applied this on the maximum temperatures of $\mathrm{Al}$ Nuzha airport station in Alexandria for year 2016 as Table 1. 
Table 1. The maximum temperatures of Al Nuzha airport station in Alexandria for year 2016

\begin{tabular}{|c|c|c|c|c|c|c|c|c|c|c|c|c|c|}
\hline & & \multicolumn{12}{|c|}{ Months } \\
\hline & & Jan & Feb & Mar & Apr & May & June & July & Aug & Sep & Oct & Nov & Dec \\
\hline \multirow{20}{*}{ Days } & 1 & 19.00 & 19.78 & 23.28 & 21.39 & 25.5 & 26.22 & 30.00 & 31.00 & 31.00 & 28.00 & 26.00 & 23.28 \\
\hline & 2 & 19.00 & 22.00 & 22.00 & 20.78 & 28.28 & 27.00 & 31.22 & 30.39 & 31.72 & 28.22 & 26.39 & 23.22 \\
\hline & 3 & 19.00 & 20.00 & 24.00 & 22.39 & 35.5 & 35.22 & 30.78 & 31.22 & 35.22 & 28.00 & 25.00 & 21.28 \\
\hline & 4 & 20.00 & 20.22 & 22.00 & 23.78 & 40.39 & 39.78 & 30.39 & 31.39 & 37.00 & 27.61 & 24.22 & 26.22 \\
\hline & 5 & 19.00 & 18.00 & 30.00 & 27.00 & 31.61 & 26.39 & 29.00 & 32.78 & 32.00 & 27.39 & 23.39 & 26.00 \\
\hline & 6 & 20.00 & 18.00 & 23.22 & 33.00 & 24.00 & 26.61 & 29.39 & 32.61 & 31.00 & 28.61 & 26.00 & 23.00 \\
\hline & 7 & 19.00 & 19.39 & 22.61 & 24.22 & 23.00 & 27.00 & 30.50 & 31.89 & 31.22 & 29.00 & 26.00 & 23.00 \\
\hline & 8 & 19.22 & 20.00 & 20.61 & 24.39 & 24.39 & 27.00 & 29.39 & 32.00 & 31.39 & 29.00 & 28.39 & 21.72 \\
\hline & 9 & 16.00 & 19.00 & 20.00 & 22.39 & 24.39 & 27.61 & 31.00 & 31.00 & 31.22 & 29.00 & 26.00 & 21.00 \\
\hline & 10 & 19.22 & 21.39 & 20.22 & 22.61 & 25.39 & 28.00 & 31.00 & 31.39 & 31.00 & 28.39 & 25.22 & 22.50 \\
\hline & 11 & 20.00 & 22.22 & 22.00 & 27.22 & 24.00 & 28.61 & 35.00 & 31.39 & 30.78 & 28.00 & 25.11 & 23.00 \\
\hline & 12 & 19.00 & 22.00 & 20.00 & 24.61 & 24.39 & 28.00 & 32.00 & 31.61 & 30.22 & 28.22 & 25.22 & 19.61 \\
\hline & 13 & 19.00 & 20.22 & 18.28 & 25.00 & 26.00 & 27.78 & 28.00 & 32.00 & 30.00 & 29.39 & 25.78 & 21.50 \\
\hline & 14 & 20.39 & 19.22 & 19.00 & 27.22 & 28.00 & 29.00 & 30.00 & 32.89 & 31.00 & 26.22 & 25.61 & 22.00 \\
\hline & 15 & 21.00 & 16.00 & 20.39 & 25.00 & 35.39 & 31.50 & 29.61 & 33.5 & 31.22 & 27.22 & 24.50 & 21.28 \\
\hline & 16 & 17.61 & 20.00 & 26.78 & 23.00 & 26.00 & 36.22 & 30.00 & 31.61 & 34.00 & 28.00 & 24.28 & 22.39 \\
\hline & 17 & 20.00 & 19.72 & 21.00 & 26.72 & 26.00 & 29.61 & 30.00 & 32.61 & 30.00 & 28.61 & 24.00 & 22.22 \\
\hline & 18 & 21.00 & 20.22 & 20.78 & 23.61 & 27.00 & 30.00 & 29.00 & 32.00 & 30.39 & 29.50 & 25.00 & 22.00 \\
\hline & 19 & 21.00 & 20.61 & 22.00 & 22.00 & 26.00 & 34.00 & 30.00 & 31.39 & 29.28 & 28.00 & 25.11 & 21.78 \\
\hline & 20 & 21.00 & 22.00 & 22.00 & 22.61 & 34.61 & 28.61 & 31.00 & 31.22 & 29.22 & 27.00 & 24.50 & 20.22 \\
\hline
\end{tabular}


Table 1. (Continued)

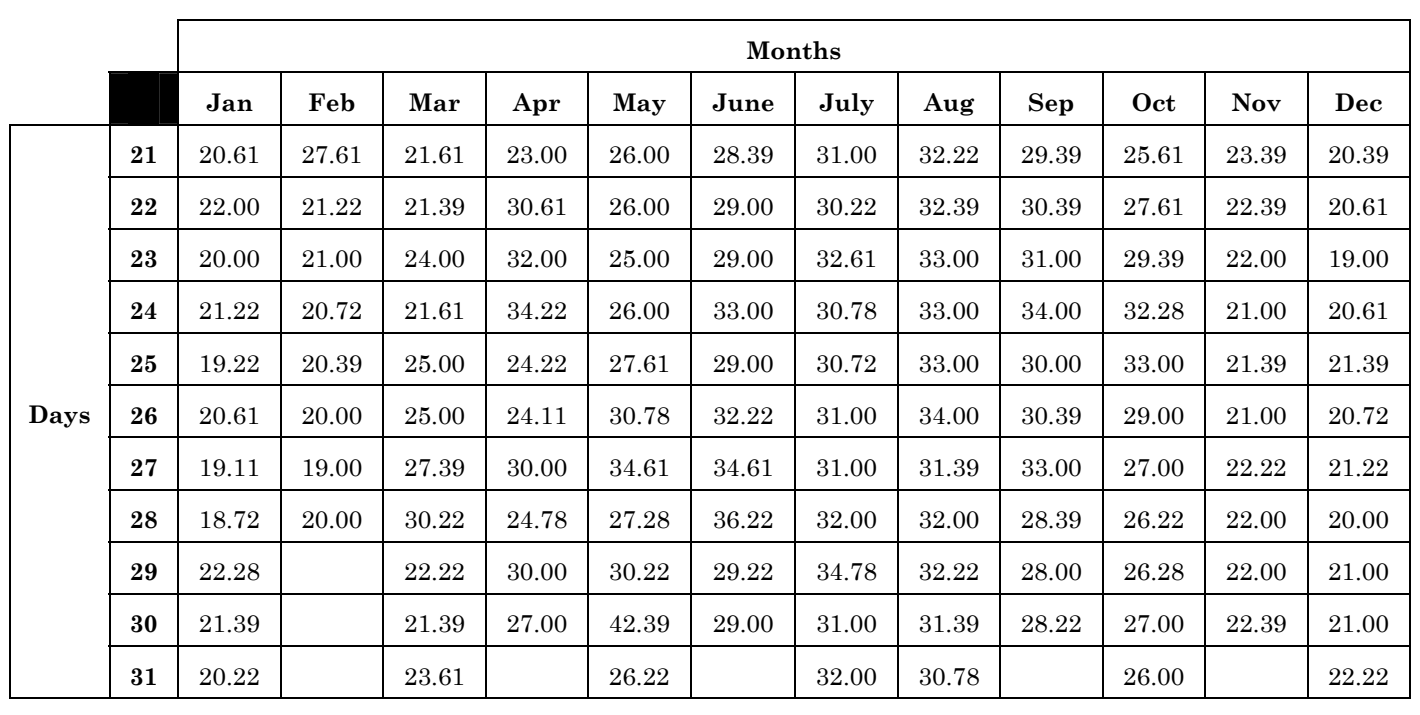




\section{Properties of the Periodogram Method}

The periodogram $I(w)$ is compute as the squared modulus of the discrete Fourier transform at frequencies $w$, and take the form

$$
I(w)=\frac{1}{n}\left|\sum_{t=1}^{n} Z(t) e^{-i w t}\right|^{2},-\pi \leq w \leq \pi
$$

where $Z(t)$ observation at time $t$. The analysis of the statistical properties of $I(w)$ is important in that it shows the poor quality of the periodogram as an estimator of the PSD and, in addition, provides some insight into how we can modify the periodogram so as to obtain better spectral estimators. We split the analysis in two parts: bias analysis and variance analysis (see Priestley [14]).

The bias and variance of an estimator are two measures often used to characterize its performance. A primary motivation is that the total squared error of the estimate is the sum of the bias squared and the variance. To see this, let a denote any quantity to be estimated, and let $\hat{a}$ be an estimate of $a$. Then the mean squared error (MSE) of the estimate is:

$$
\begin{aligned}
\operatorname{MSE}(\hat{a}) \cong & E\left\{|\hat{a}-a|^{2}\right\} \\
= & E\left\{|\hat{a}-E\{\hat{a}\}+E\{\hat{a}\}-a|^{2}\right\} \\
= & E\left\{|\hat{a}-E\{\hat{a}\}|^{2}\right\}+|E\{\hat{a}\}-a|^{2} \\
& +2 \operatorname{Re}[E\{\hat{a}-E\{\hat{a}\}\} \cdot(E\{\hat{a}\}-a)] \\
= & \operatorname{Var}(\hat{a})+\mid \text { bais }\left.\{\hat{a}\}\right|^{2} .
\end{aligned}
$$

By separately considering the bias and variance components of the MSE as following: 


\subsection{Bias analysis of the periodogram}

Since the expected value of the periodogram is defined (Stoica and Moses [69]) as:

$$
E(I(w))=\sum_{k=-(n-1)}^{n-1} E\left(\hat{\gamma}_{k}\right) e^{-i w k}
$$

where $\hat{\gamma}_{k}$ denotes an estimate of the autocovariance sequence $\gamma_{k}$ and defined as:

$$
\hat{\gamma}_{k}=\frac{1}{n} \sum_{t=k+1}^{n} Z_{t} Z_{t-k}^{*}, \quad 0 \leq k \leq n-1,
$$

then

$$
E\left\{\hat{\gamma}_{k}\right\}=\left(1-\frac{K}{n}\right) \gamma_{k}, \quad K \geq 0
$$

and

$$
E\left\{\hat{\gamma}_{-k}\right\}=\left(1-\frac{K}{n}\right) \gamma_{-k},-K \leq 0
$$

hence

$$
E(I(w))=\sum_{k=-(n-1)}^{n-1}\left(1-\frac{|K|}{n}\right) \gamma_{k} e^{-i w k}
$$

Define

$$
w_{B}(k)=\left\{\begin{array}{cc}
1-\frac{|K|}{n}, & k=0, \pm 1, \pm 2, \ldots, \pm(n-1) \\
0, & \text { otherwise. }
\end{array}\right.
$$

The sequence (8) is called triangular window, or the Bartlett window. By using $w_{B}(k)$, we can write (7) as a DTFT:

$$
E\{I(w)\}=\sum_{k=-\infty}^{\infty}\left[w_{B}(k) \gamma_{k}\right] e^{-i w k}
$$


The DTFT of the product of two sequences is equal to the convolution of their respective DTFTs. Hence, (9) leads to

$$
E(I(w))=\frac{1}{2 \pi} \int_{-\pi}^{\pi} \varnothing(\psi) W_{B}(w-\psi) d \psi
$$

where $W_{B}(w)$ is the DTFT of the triangular window and $\varnothing(\psi)$ is spectral density function as mentioned in the first chapter. Previously, it has been concluded that this periodogram is asymptotically (for large $n$ ) unbiased, this can be expressed mathematically by

$$
\lim _{n \rightarrow \infty} E(I(w))=\varnothing(w) .
$$

For more details, please refer to (Stoica and Moses [15]).

\subsection{Covariance analysis of the periodogram}

The finite-sample variance of $I(w)$ can be easily established only in some specific cases, such as in the case of Gaussian white noise. The asymptotic variance of $I(w)$, however, can be derived for more general signals. In the following, we present an asymptotic (for $n \geq 1$ ) analysis of the variance of $I(w)$ since it turns out to be sufficient for showing the poor statistical accuracy of the periodogram (for a finite sample analysis). Some preliminary discussion is required. A sequence $\{e(t)\}$ is called complex (or circular) white noise if it satisfies

$$
E\left\{e(t) e^{*}(s)\right\}=\sigma^{2} \delta_{t, s},
$$

and

$$
E\{e(t) e(s)\}=0, \text { for all } t \text { and } s
$$


Note that $\sigma^{2}=E\left\{|e(t)|^{2}\right\}$ is the variance of $e(t)$. Equation (12) can be rewritten as

$$
\left\{\begin{array}{c}
E\{\operatorname{Re}[e(t)] \operatorname{Re}[e(s)]\}=\frac{\sigma^{2}}{2} \delta_{t, s}, \\
E\{\operatorname{Im}[e(t)] \operatorname{Im}[e(s)]\}=\frac{\sigma^{2}}{2} \delta_{t, s}, \\
E\{\operatorname{Re}[e(t)] \operatorname{Im}[e(s)]\}=0 .
\end{array}\right.
$$

Hence, the real and imaginary parts of a complex white noise are realvalued white noise sequences of identical power equal to $\sigma^{2} / 2$, and uncorrelated with one another. In what follows, we shall also make use of the symbol $\widehat{O}\left(1 / n^{\alpha}\right)$, for some $\alpha>0$, to denote a random variable which is such that the square root of its second-order moment goes to zero at least as fast as $1 / n^{\alpha}$, as $n$ tends to infinity. The asymptotic variance/ covariance of $I(w)$ in the general case are:

$$
\begin{array}{r}
\lim _{n \rightarrow \infty} E\left\{\left[I\left(w_{1}\right)-E\left(I\left(w_{1}\right)\right)\right]\left[I\left(w_{2}\right)-E\left(I\left(w_{2}\right)\right)\right]\right. \\
= \begin{cases}\varnothing^{2}\left(w_{1}\right), & w_{1}=w_{2}, \\
0, & w_{1} \neq w_{2} .\end{cases}
\end{array}
$$

Previously it has been concluded that this periodogram has a large variance, even for large $n$. For more details, please refer to (Stoica and Moses [15]).

\section{Proposed Method}

In this section, we present a method [16] for Chen to convert the ordinary time series into fuzzy time series and forecasting observation for the last series. This method aimed to attain better forecasting accuracy by using fuzzy time series and summarized in the following six steps: 
(1) Define the universe of discourse and partition it into equally lengthy intervals.

(2) Calculate the number of observations in each interval, and by doing so, there will be a re-division for each interval based on the number of observations contained in these interval.

(3) Define linguistic values represented by fuzzy set $A_{i}$ based on the re-divided intervals.

(4) Fuzzify the actual observations.

(5) Identify and establish fuzzy logical relationships based on the fuzzified observations.

(6) Use set of rules to determine whether the trend of the forecasting goes up or down, this mean we defuzzify the fuzzy output into forecasted output.

\section{Stages of Building Simulation Experiments}

Depending on the program, the simulation experiments for this part consists of seven stages.

(1) Stage of choosing the default parameters values by randomly select both of the sample sizes $n$ and the default values for frequencies $w$.

(2) Stage of bringing random observations from our life which is a realizations of a process but it is not easy to find it. We bringing here the maximum temperatures of $\mathrm{Al}$ Nuzha airport station in Alexandria for year 2016 as shown in Table 1.

(3) Depending on the size of the sample, we randomly take some actual observations in the previous step and transform them to fuzzy observations. Defuzzify the fuzzy observations into forecasted observations which carrying numerical values. This transformation and defuzzification are completed by Chen [16]. 
(4) Substituting by both types of observations in Equation (1) to obtain the periodogram in the case of actual observations, which is symbolized by $I_{1}(w)$ and in the case of the forecasted observations, which is symbolized by $I_{2}(w)$.

(5) Study the statistical properties which are the bias and the variance of both $I_{1}(w)$ and $I_{2}(w)$.

(6) Compare between $I_{1}(w)$ and $I_{2}(w)$ by using the MSE which depends on the statistical properties.

(7) Repeat this comparison a lot with another sample sizes and with change of the default values for frequencies.

\section{Creating Program for the Large Sample Size}

Because of the time series maybe deals with large size samples and takes into account the length of the proposed method, we created program implemented this proposed method for the large sample size as hundreds, thousands or millions. Also use this program to apply simulation experiments. 


\section{The Results}

Table 2. The periodogram in case of actual observations and forecasted observations

\begin{tabular}{|c|c|c|c|c|c|c|c|}
\hline$n$ & $w$ & $I_{1}(w)$ & $I_{2}(w)$ & $n$ & $w$ & $I_{1}(w)$ & $I_{2}(w)$ \\
\hline \multirow{10}{*}{50} & 0.02 & 170.35819 & 134.82559 & \multirow{10}{*}{250} & 0.02 & 29.900976 & 29.135670 \\
\hline & 0.009 & 706.53464 & 708.50856 & & 0.009 & 112.75412 & 112.69812 \\
\hline & 0.03 & 66.333260 & 65.758763 & & 0.03 & 10.796615 & 10.685451 \\
\hline & 0.01 & 683.46336 & 686.85087 & & 0.01 & 154.12567 & 152.65525 \\
\hline & 0.004 & 2107.6057 & 2112.8801 & & 0.004 & 938.27178 & 936.55098 \\
\hline & 0.007 & 1595.1627 & 1609.2737 & & 0.007 & 286.89489 & 272.98740 \\
\hline & 0.08 & 13.156406 & 13.094207 & & 0.08 & 0.9794824 & 0.9734677 \\
\hline & 0.06 & 6.5554032 & 6.5023031 & & 0.06 & 2.0878158 & 2.0975654 \\
\hline & 0.05 & 13.156474 & 13.094223 & & 0.05 & 1.4669779 & 1.4546001 \\
\hline & 0.07 & 4.4869086 & 4.4707815 & & 0.07 & 2.1061621 & 2.0518602 \\
\hline \multirow{10}{*}{120} & 0.02 & 83.221004 & 83.430600 & \multirow{10}{*}{310} & 0.02 & 2.1061612 & 2.0518656 \\
\hline & 0.009 & 221.75856 & 218.17692 & & 0.009 & 150.23381 & 149.87430 \\
\hline & 0.03 & 25.532098 & 24.927336 & & 0.03 & 7.8455078 & 7.7762468 \\
\hline & 0.01 & 683.46330 & 686.85080 & & 0.01 & 121.86160 & 120.45476 \\
\hline & 0.004 & 1800.4151 & 1744.5509 & & 0.004 & 818.90386 & 814.34466 \\
\hline & 0.007 & 1595.1627 & 1609.2737 & & 0.007 & 261.42247 & 258.86507 \\
\hline & 0.08 & 1.1639950 & 1.1696846 & & 0.08 & 0.7119543 & 0.7162692 \\
\hline & 0.06 & 2.6701001 & 2.6880069 & & 0.06 & 1.3778661 & 1.4110343 \\
\hline & 0.05 & 4.6687745 & 4.6221076 & & 0.05 & 1.9238701 & 1.8791745 \\
\hline & 0.07 & 2.3167680 & 2.2773975 & & 0.07 & 1.4343323 & 1.4370867 \\
\hline
\end{tabular}


Table 2. (Continued)

\begin{tabular}{|c|c|c|c|c|c|c|c|}
\hline$n$ & $w$ & $I_{1}(w)$ & $I_{2}(w)$ & $n$ & $w$ & $I_{1}(w)$ & $I_{2}(w)$ \\
\hline \multirow{10}{*}{180} & 0.02 & 44.525100 & 44.991901 & \multirow{10}{*}{365} & 0.02 & 22.036070 & 21.442731 \\
\hline & 0.009 & 238.15376 & 238.25316 & & 0.009 & 119.56787 & 118.68913 \\
\hline & 0.03 & 12.743045 & 12.551134 & & 0.03 & 9.6866210 & 9.6240370 \\
\hline & 0.01 & 181.86370 & 181.66016 & & 0.01 & 96.398410 & 97.428834 \\
\hline & 0.004 & 974.86659 & 974.29167 & & 0.004 & 703.96809 & 696.79300 \\
\hline & 0.007 & 448.98284 & 455.87200 & & 0.007 & 200.7328 & 201.87159 \\
\hline & 0.08 & 0.4488206 & 0.4509409 & & 0.08 & 0.5133610 & 0.5080475 \\
\hline & 0.06 & 2.4200968 & 2.3885979 & & 0.06 & 1.340107 & 1.3490445 \\
\hline & 0.05 & 4.8805215 & 4.9670604 & & 0.05 & 2.939900 & 2.9147945 \\
\hline & 0.07 & 2.0315685 & 2.0340340 & & 0.07 & 0.8918780 & 0.8948026 \\
\hline
\end{tabular}

One can see that:

(I) From Table 3, the properties for each periodogram are achieved and are, whenever the sample size increased, each periodogram has less bias and large variance. 
Table 3. The statistical properties for $I_{1}(w)$ and $I_{2}(w)$

\begin{tabular}{|c|c|c|c|c|c|}
\hline$n$ & $w$ & $\operatorname{Bias}\left(I_{1}(w)\right)$ & $\operatorname{Bias}\left(I_{2}(w)\right)$ & $\operatorname{Var}\left(I_{1}(w)\right)$ & $\operatorname{Var}\left(I_{2}(w)\right)$ \\
\hline \multirow{10}{*}{50} & 0.02 & -224557202.981 & -200091291.760 & $5.0425937657 \mathrm{e}+16$ & $4.0036525968 \mathrm{e}+16$ \\
\hline & 0.009 & -656894.326104 & -654912.62493 & 431510155759.900 & 428910545086.960 \\
\hline & 0.03 & -7103483098.99 & -7069851571.00 & $5.045947295 \mathrm{e}+19$ & $4.9982801567 \mathrm{e}+19$ \\
\hline & 0.01 & -525393.716107 & -522131.492994 & 276038558018.985 & 272621295232.064 \\
\hline & 0.004 & -88513.1139124 & -88296.6119801 & 7834571310.02340 & 7796291646.06844 \\
\hline & 0.007 & -142193.850029 & -141797.602325 & 20219085064.0954 & 20106566682.0654 \\
\hline & 0.08 & $-4.7993412 \mathrm{e}+23$ & $-4.7392040 \mathrm{e}+23$ & $2.3033694023 \mathrm{e}+47$ & $2.2460055094 \mathrm{e}+47$ \\
\hline & 0.06 & $-1.5625907 \mathrm{e}+18$ & $-1.5469760 \mathrm{e}+18$ & $2.4416887760 \mathrm{e}+36$ & $2.3930558523 \mathrm{e}+36$ \\
\hline & 0.05 & $-5.7256706 \mathrm{e}+18$ & $-5.6789678 \mathrm{e}+18$ & $3.2783395672 \mathrm{e}+37$ & $3.2249990847 \mathrm{e}+37$ \\
\hline & 0.07 & $-2.4245668 \mathrm{e}+22$ & $-2.3858168 \mathrm{e}+22$ & $5.8785303125 \mathrm{e}+44$ & $5.6921344663 \mathrm{e}+44$ \\
\hline \multirow{10}{*}{120} & 0.02 & $-3.5505896 \mathrm{e}+15$ & $-3.5024568 \mathrm{e}+15$ & $1.2606633823 \mathrm{e}+31$ & $1.2267157447 \mathrm{e}+31$ \\
\hline & 0.009 & -9157262358.04 & -9067727911.96 & $8.3855453832 \mathrm{e}+19$ & $8.2223689466 \mathrm{e}+19$ \\
\hline & 0.03 & $-8.7279759 e+21$ & $-8.6890388 \mathrm{e}+21$ & $7.6177562257 \mathrm{e}+43$ & $7.5499398293 \mathrm{e}+43$ \\
\hline & 0.01 & -1867793320.34 & -1864548872.95 & $3.4886518856 \mathrm{e}+18$ & $3.4765424912 \mathrm{e}+18$ \\
\hline & 0.004 & -1074513.00503 & -1057174.35016 & 1154579268506.96 & 1117617535623.00 \\
\hline & 0.007 & -39830787.6043 & -39661359.3056 & $1.5864916687 \mathrm{e}+15$ & $1.573023403 \mathrm{e}+15$ \\
\hline & 0.08 & $-1.5992230 \mathrm{e}+67$ & $-1.5960070 \mathrm{e}+67$ & $2.557515878 \mathrm{e}+134$ & $2.547239791 \mathrm{e}+134$ \\
\hline & 0.06 & $-3.0362189 \mathrm{e}+41$ & $-3.0109025 \mathrm{e}+41$ & $9.2186242696 \mathrm{e}+82$ & $9.0655313641 \mathrm{e}+82$ \\
\hline & 0.05 & $-1.0416784 \mathrm{e}+40$ & $-1.0317544 \mathrm{e}+40$ & $1.0850943216 \mathrm{e}+80$ & $1.0645179414 \mathrm{e}+80$ \\
\hline & 0.07 & $-1.585075 \mathrm{e}+48$ & $-1.5753440 \mathrm{e}+48$ & $2.5124646525 \mathrm{e}+96$ & $2.4817088478 \mathrm{e}+96$ \\
\hline \multirow{10}{*}{180} & 0.02 & $-1.1545569 \mathrm{e}+22$ & $-1.1334863 e+22$ & $1.3330016655 \mathrm{e}+44$ & $1.2847912728 \mathrm{e}+44$ \\
\hline & 0.009 & -389973235721 & -388305826844 & $1.5207912454 \mathrm{e}+23$ & $1.5078141517 \mathrm{e}+23$ \\
\hline & 0.03 & $-1.1858283 \mathrm{e}+34$ & $-1.1682750 \mathrm{e}+34$ & $1.4061888013 \mathrm{e}+68$ & $1.3648665446 \mathrm{e}+68$ \\
\hline & 0.01 & -3465297794158 & -3462772765086 & $1.2008288801 \mathrm{e}+25$ & $1.1990795226 \mathrm{e}+25$ \\
\hline & 0.004 & -96068332.9404 & -95618874.0045 & $9.2291244711 \mathrm{e}+15$ & $9.1429691206 \mathrm{e}+15$ \\
\hline & 0.007 & -6571255367.03 & -6568378754.59 & $4.3181397093 \mathrm{e}+19$ & $4.3143599467 \mathrm{e}+19$ \\
\hline & 0.08 & $-7.374350 \mathrm{e}+112$ & $-7.365770 \mathrm{e}+112$ & $5.438105592 \mathrm{e}+225$ & $5.425467027 \mathrm{e}+225$ \\
\hline & 0.06 & $-5.2982047 \mathrm{e}+61$ & $-5.2424564 \mathrm{e}+61$ & $2.807097394 \mathrm{e}+123$ & $2.748307283 \mathrm{e}+123$ \\
\hline & 0.05 & $-5.3017752 \mathrm{e}+51$ & $-5.2413556 e+51$ & $2.810882094 \mathrm{e}+103$ & $2.747180886 \mathrm{e}+103$ \\
\hline & 0.07 & $-5.2388512 \mathrm{e}+71$ & $-5.0640549 e+71$ & $2.744559524 \mathrm{e}+143$ & $2.564465247 \mathrm{e}+143$ \\
\hline
\end{tabular}


USING THE FUZZY TIME SERIES TECHNIQUE TO ...

Table 3. (Continued)

\begin{tabular}{|c|c|c|c|c|c|}
\hline$n$ & $w$ & $\operatorname{Bias}\left(I_{1}(w)\right)$ & $\operatorname{Bias}\left(I_{2}(w)\right)$ & $\operatorname{Var}\left(I_{1}(w)\right)$ & $\operatorname{Var}\left(I_{2}(w)\right)$ \\
\hline \multirow{10}{*}{250} & 0.02 & $-6.7970150 \mathrm{e}+29$ & $-6.7362127 \mathrm{e}+29$ & $4.6199414144 \mathrm{e}+59$ & $4.5376562866 \mathrm{e}+59$ \\
\hline & 0.009 & $-5.4694453 \mathrm{e}+17$ & $-5.4272701 \mathrm{e}+17$ & $2.9914831966 \mathrm{e}+35$ & $2.9455261515 \mathrm{e}+35$ \\
\hline & 0.03 & $-3.7551217 \mathrm{e}+43$ & $-3.6687603 e+43$ & $1.4100939285 \mathrm{e}+87$ & $1.3459802725 \mathrm{e}+87$ \\
\hline & 0.01 & $-2.1144902 \mathrm{e}+16$ & $-2.1015432 \mathrm{e}+16$ & $4.4710689754 \mathrm{e}+32$ & $4.4164842274 \mathrm{e}+32$ \\
\hline & 0.004 & -452890930.054 & -452164010.982 & $2.0511019504 \mathrm{e}+17$ & $2.0445229243 \mathrm{e}+17$ \\
\hline & 0.007 & -2485300482977 & -2423037390157 & $6.1767184905 \mathrm{e}+24$ & $5.8711101942 \mathrm{e}+24$ \\
\hline & 0.08 & $-2.413215 \mathrm{e}+113$ & $-2.357223 \mathrm{e}+113$ & $5.823615974 \mathrm{e}+226$ & $5.556494464 \mathrm{e}+226$ \\
\hline & 0.06 & $-1.9678505 \mathrm{e}+85$ & $-1.9435608 \mathrm{e}+85$ & $3.872470163 \mathrm{e}+170$ & $3.777428951 \mathrm{e}+170$ \\
\hline & 0.05 & $-2.7371720 \mathrm{e}+87$ & $-2.7148932 \mathrm{e}+87$ & $7.492111423 \mathrm{e}+174$ & $7.370645545 \mathrm{e}+174$ \\
\hline & 0.07 & $-2.5271875 \mathrm{e}+99$ & $-2.4971292 \mathrm{e}+99$ & $6.386676723 \mathrm{e}+198$ & $6.235656316 \mathrm{e}+198$ \\
\hline \multirow{10}{*}{310} & 0.02 & $-3.1964833 \mathrm{e}+36$ & $-3.1600646 \mathrm{e}+36$ & $1.0217506004 \mathrm{e}+73$ & $9.9860087475 \mathrm{e}+72$ \\
\hline & 0.009 & $-9.590586 \mathrm{e}+17$ & $-9.5272425 \mathrm{e}+17$ & $9.1979341593 \mathrm{e}+35$ & $9.0768350106 \mathrm{e}+35$ \\
\hline & 0.03 & $-4.7235960 \mathrm{e}+53$ & $-4.6945753 \mathrm{e}+53$ & $2.231236005 \mathrm{e}+107$ & $2.203903776 \mathrm{e}+107$ \\
\hline & 0.01 & $-4.2875181 \mathrm{e}+19$ & $-4.2468007 \mathrm{e}+19$ & $1.8382811615 \mathrm{e}+39$ & $1.8035316877 \mathrm{e}+39$ \\
\hline & 0.004 & -8449197625.95 & -8440890732.94 & $7.1388940518 \mathrm{e}+19$ & $7.1248636356 \mathrm{e}+19$ \\
\hline & 0.007 & $-4.7825890 \mathrm{e}+10$ & $-4.7196455 e+10$ & $2.2873156136 \mathrm{e}+29$ & $2.2275041505 \mathrm{e}+29$ \\
\hline & 0.08 & $-2.302463 e+140$ & $-2.275953 \mathrm{e}+140$ & $5.301340316 \mathrm{e}+280$ & $5.179965604 \mathrm{e}+280$ \\
\hline & 0.06 & $-2.898353 e+105$ & $-2.822471 \mathrm{e}+105$ & $8.400454206 \mathrm{e}+210$ & $7.966344824 \mathrm{e}+210$ \\
\hline & 0.05 & $-1.3996074 \mathrm{e}+88$ & $-1.3875873 e+88$ & $1.958901157 \mathrm{e}+176$ & $1.925398655 \mathrm{e}+176$ \\
\hline & 0.07 & $-1.062746 \mathrm{e}+123$ & $-1.050332 \mathrm{e}+123$ & $1.129429306 \mathrm{e}+246$ & $1.103198905 \mathrm{e}+246$ \\
\hline \multirow{10}{*}{365} & 0.02 & $-4.3695133 \mathrm{e}+42$ & $-4.3233904 \mathrm{e}+42$ & $1.9092647045 \mathrm{e}+85$ & $1.8691705126 \mathrm{e}+85$ \\
\hline & 0.009 & $-4.6992576 \mathrm{e}+20$ & $-4.6982559 \mathrm{e}+20$ & $2.2083022400 \mathrm{e}+41$ & $2.2073608606 \mathrm{e}+41$ \\
\hline & 0.03 & $-9.0300037 \mathrm{e}+62$ & $-9.0192242 \mathrm{e}+62$ & $8.154096736 \mathrm{e}+125$ & $8.134640555 \mathrm{e}+125$ \\
\hline & 0.01 & $-4.6046619 \mathrm{e}+22$ & $-4.4041645 e+22$ & $2.1202911255 \mathrm{e}+45$ & $1.9396665287 \mathrm{e}+45$ \\
\hline & 0.004 & -136150186382 & -135044170675 & $1.8536873254 \mathrm{e}+22$ & $1.8236928037 \mathrm{e}+22$ \\
\hline & 0.007 & $-5.911374 \mathrm{e}+16$ & $-5.9110935 e+16$ & $3.4944349532 \mathrm{e}+33$ & $3.4941020871 \mathrm{e}+33$ \\
\hline & 0.08 & $-4.251415 \mathrm{e}+152$ & $-4.229428 \mathrm{e}+152$ & $1.807453025 \mathrm{e}+305$ & $1.788806866 \mathrm{e}+305$ \\
\hline & 0.06 & $-1.344969 \mathrm{e}+124$ & $-1.325507 \mathrm{e}+124$ & $1.808942415 \mathrm{e}+248$ & $1.756969275 \mathrm{e}+248$ \\
\hline & 0.05 & $-4.356043 \mathrm{e}+103$ & $-3.211722 \mathrm{e}+103$ & $1.897511227 \mathrm{e}+207$ & $1.031516223 \mathrm{e}+207$ \\
\hline & 0.07 & $-3.480709 \mathrm{e}+144$ & $-3.474175 \mathrm{e}+144$ & $1.211533806 \mathrm{e}+289$ & $1.206989423 \mathrm{e}+289$ \\
\hline
\end{tabular}


(II) From Table 4, the MSE for $I_{2}(w)$ less than the MSE for $I_{1}(w)$ along this table. Through these notes, we find that $I_{2}(w)$ is better than $I_{1}(w)$ as an estimators for the spectral density function and which means the estimation improvement by the fuzzy time series method. 
Table 4. The MSEs for $I_{1}(w)$ and $I_{2}(w)$

\begin{tabular}{|c|c|c|c|c|c|c|c|}
\hline$n$ & $w$ & $M S E\left(I_{1}(w)\right)$ & $M S E\left(I_{2}(w)\right)$ & $n$ & $w$ & $M S E\left(I_{1}(w)\right)$ & $\operatorname{MSE}\left(I_{2}(w)\right)$ \\
\hline \multirow{10}{*}{50} & 0.02 & $1.00851805 \mathrm{e}+17$ & $8.00730500 \mathrm{e}+16$ & \multirow{10}{*}{250} & 0.02 & $9.23988282 \mathrm{e}+59$ & $9.07531257 \mathrm{e}+59$ \\
\hline & 0.009 & 863020311519 & 857821090173 & & 0.009 & $5.98296639 \mathrm{e}+35$ & $5.89105230 \mathrm{e}+35$ \\
\hline & 0.03 & $1.00918946 \mathrm{e}+20$ & $9.99656020 \mathrm{e}+19$ & & 0.03 & $2.82018780 \mathrm{e}+87$ & $2.69196054 \mathrm{e}+87$ \\
\hline & 0.01 & 552077116036 & 545242590464 & & 0.01 & $8.94213795 \mathrm{e}+32$ & $8.83296845 \mathrm{e}+32$ \\
\hline & 0.004 & 15669142620 & 15592583293 & & 0.004 & $4.10220390 \mathrm{e}+17$ & $4.08904584 \mathrm{e}+17$ \\
\hline & 0.007 & 40438170128 & 40213133364 & & 0.007 & $1.23534369 \mathrm{e}+25$ & $1.17422203 \mathrm{e}+25$ \\
\hline & 0.08 & $4.60673896 \mathrm{e}+47$ & $4.492011006 \mathrm{e}+47$ & & 0.08 & $1.1647231 \mathrm{e}+227$ & $1.1112988 \mathrm{e}+227$ \\
\hline & 0.06 & $4.88337755 \mathrm{e}+36$ & $4.78611176 \mathrm{e}+36$ & & 0.06 & $7.7449403 \mathrm{e}+170$ & $7.5548579 \mathrm{e}+170$ \\
\hline & 0.05 & $6.55667908 \mathrm{e}+37$ & $6.44999813 \mathrm{e}+37$ & & 0.05 & $1.4984225 \mathrm{e}+175$ & $1.4741291 \mathrm{e}+175$ \\
\hline & 0.07 & $1.17570607 \mathrm{e}+45$ & $1.13842687 \mathrm{e}+45$ & & 0.07 & $1.2773353 \mathrm{e}+199$ & $1.2471312 \mathrm{e}+199$ \\
\hline \multirow{10}{*}{120} & 0.02 & $1.52355124 \mathrm{e}+44$ & $1.50998796 \mathrm{e}+44$ & \multirow{10}{*}{310} & 0.02 & $2.04350120 \mathrm{e}+73$ & $1.99720174 \mathrm{e}+73$ \\
\hline & 0.009 & $1.67710907 \mathrm{e}+20$ & $1.64447378 \mathrm{e}+20$ & & 0.009 & $1.83958683 \mathrm{e}+36$ & $1.81536700 \mathrm{e}+36$ \\
\hline & 0.03 & $1.25109002 \mathrm{e}+50$ & $1.22650352 \mathrm{e}+50$ & & 0.03 & $4.4624720 \mathrm{e}+107$ & $4.4078075 \mathrm{e}+107$ \\
\hline & 0.01 & $6.97730377 \mathrm{e}+18$ & $6.95308499 \mathrm{e}+18$ & & 0.01 & $3.67656232 \mathrm{e}+39$ & $3.60706337 \mathrm{e}+39$ \\
\hline & 0.004 & 2309158537012 & 2235235071247 & & 0.004 & $1.42777881 \mathrm{e}+20$ & $1.42497272 \mathrm{e}+20$ \\
\hline & 0.007 & $3.17298333 \mathrm{e}+15$ & $3.14604680 \mathrm{e}+15$ & & 0.007 & $4.57463125 \mathrm{e}+29$ & $4.45500830 \mathrm{e}+29$ \\
\hline & 0.08 & $5.1150317 \mathrm{e}+134$ & $5.0944795 \mathrm{e}+134$ & & 0.08 & $1.0602680 \mathrm{e}+281$ & $1.0359931 \mathrm{e}+281$ \\
\hline & 0.06 & $1.84372485 \mathrm{e}+83$ & $1.81310627 \mathrm{e}+83$ & & 0.06 & $1.6800908 \mathrm{e}+211$ & $1.5932689 \mathrm{e}+211$ \\
\hline & 0.05 & $2.17018864 \mathrm{e}+80$ & $2.12903588 \mathrm{e}+80$ & & 0.05 & $3.9178023 \mathrm{e}+176$ & $3.8507973 \mathrm{e}+176$ \\
\hline & 0.07 & $5.02492930 \mathrm{e}+96$ & $4.96341769 \mathrm{e}+96$ & & 0.07 & $2.2588586 \mathrm{e}+246$ & $2.2063978 \mathrm{e}+246$ \\
\hline
\end{tabular}


Table 4. (Continued)

\begin{tabular}{|c|c|c|c|c|c|c|c|}
\hline$n$ & $w$ & $M S E\left(I_{1}(w)\right)$ & $\operatorname{MSE}\left(I_{2}(w)\right)$ & $n$ & $w$ & $M S E\left(I_{1}(w)\right)$ & $M S E\left(I_{2}(w)\right)$ \\
\hline \multirow{10}{*}{180} & 0.02 & $2.66600333 \mathrm{e}+44$ & $2.56958254 \mathrm{e}+44$ & \multirow{10}{*}{365} & 0.02 & $3.81852940 \mathrm{e}+85$ & $3.73834102 \mathrm{e}+85$ \\
\hline & 0.009 & $3.04158249 \mathrm{e}+23$ & $3.01562830 \mathrm{e}+23$ & & 0.009 & $4.41660448 \mathrm{e}+41$ & $4.41472172 \mathrm{e}+41$ \\
\hline & 0.03 & $2.81237760 \mathrm{e}+68$ & $2.72973308 \mathrm{e}+68$ & & 0.03 & $1.6308193 \mathrm{e}+126$ & $1.6269281 \mathrm{e}+126$ \\
\hline & 0.01 & $2.40165776 \mathrm{e}+25$ & $2.39815904 \mathrm{e}+25$ & & 0.01 & $4.24058225 \mathrm{e}+45$ & $3.87933305 \mathrm{e}+45$ \\
\hline & 0.004 & $1.84582489 \mathrm{e}+16$ & $1.82859382 \mathrm{e}+16$ & & 0.004 & $3.70737465 \mathrm{e}+22$ & $3.64738560 \mathrm{e}+22$ \\
\hline & 0.007 & $8.63627941 \mathrm{e}+19$ & $8.62871989 \mathrm{e}+19$ & & 0.007 & $6.98886990 \mathrm{e}+33$ & $6.98820416 \mathrm{e}+33$ \\
\hline & 0.08 & $1.0876211 \mathrm{e}+226$ & $1.0850934 \mathrm{e}+226$ & & 0.08 & $3.6149060 \mathrm{e}+305$ & $3.5776137 \mathrm{e}+305$ \\
\hline & 0.06 & $5.6141947 \mathrm{e}+123$ & $5.4966145 \mathrm{e}+123$ & & 0.06 & $3.6178848 \mathrm{e}+248$ & $3.5139385 \mathrm{e}+248$ \\
\hline & 0.05 & $5.6217641 \mathrm{e}+103$ & $5.4943617 \mathrm{e}+103$ & & 0.05 & $3.7950224 \mathrm{e}+207$ & $2.0630324 \mathrm{e}+207$ \\
\hline & 0.07 & $5.4891190 \mathrm{e}+143$ & $5.1289304 \mathrm{e}+143$ & & 0.07 & $2.4230676 \mathrm{e}+289$ & $2.4139788 \mathrm{e}+289$ \\
\hline
\end{tabular}




\section{Conclusion}

The fuzzy time series technique is applied to realistic real observations. We got the estimator of spectral density function "periodogram" in case of real observations and forecasted observations of the fuzzy time series. The statistical properties of this estimator are studied. It is noted from Table 4 that: the fuzzy time series technique improves the estimation using the spectral analysis based on the mean square errors.

\section{References}

[1] G. G. Stokes, Note on searching for periodicities, Proceedings of the Royal Society 29 (1879), pp. 122.

[2] A. Schuster, On the investigation of hidden periodicities with application to a supposed 26 day period of meteorological phenomena, Journal of Geophysical Research 3(1) (1898), 13-41.

$$
\text { DOI: https://doi.org/10.1029/TM003i001p00013 }
$$

[3] R. B. Blackman and J. W. Tukey, The measurement of power spectra from the point of view of communications engineering-Part I, Bell Labs Technical Journal 37(1) (1958), 185-282.

DOI: https://doi.org/10.1002/j.1538-7305.1958.tb03874.x

[4] J. W. Cooley and J. W. Tukey, An algorithm for the machine calculation of complex Fourier series, Mathematics of Computation 19(90) (1965), 297-301.

DOI: https://doi.org/10.1090/S0025-5718-1965-0178586-1

[5] T. C. M. Lee, A simple span selector for periodogram smoothing, Biometrika 84(4) (1997), 965-969.

$$
\text { DOI: https://doi.org/10.1093/biomet/84.4.965 }
$$

[6] E. Parzen, On spectral analysis with missing observations and amplitude modulation, Sankhyā: The Indian Journal of Statistics, Series A 25(4) (1963), 383-392.

[7] T. W. Anderson, The Statistical Analysis of Time Series, John Wiley \& Sons, New York, 1971.

[8] M. M. Mohie El-Din, A. A. M. Teamah and H. M. Faied, Asymptotic statistical properties of spectral estimates with different tapers for discrete short time processes, International Journal of Mathematics \& Computation 3(J09) (2009), $133-145$. 
[9] M. M. Mohie El-Din, A. A. M. Teamah and H. M. Faied, Multitaper multidimensional spectral estimators for a discrete time process with missed observations, International Journal of Mathematics \& Computation 7(J10) (2010), 61-72.

[10] A. A. M. Teamah and H. S. Bakouch, A statistical study on some spectral estimators with different multitapers for continuous-time complex processes, International Journal of Applied Mathematics 15 (2004), 253-270.

[11] A. A. M. Teamah and H. S. Bakouch, Asymptotic statistical properties of spectral estimates with different tapers for discrete time processes, Applied Mathematics and Computation 150(3) (2004), 681-695.

DOI: https://doi.org/10.1016/S0096-3003(03)00299-6

[12] A. A. M. Teamah and H. S. Bakouch, Multitaper multidimensional spectral estimators for a continuous time process with missed observations, International Journal of Applied Mathematics and Statistics 3(M05) (2005), 87-104.

[13] A. A. M. Teamah and H. S. Bakouch, Statistical analysis on the average of periodograms with different tapers and spectral estimates of continuous time process, Advances in Modeling and Analysis D 8(1/2) (2003), 62-81.

[14] M. B. Priestley, Spectral Analysis and Time Series, London, England: Academic Press, 1981.

[15] P. Stoica and R. L. Moses, Spectral Analysis of Signals (Vol. 452), Upper Saddle River, NJ: Pearson Prentice Hall, 2005.

[16] S. M. Chen and C. C. Hsu, A new method to forecast enrollments using fuzzy time series, International Journal of Applied Science and Engineering 2(3) (2004), 234-244. 